\title{
Pelvic actinomycosis presenting as ovarian neoplasia: a case report
}

\author{
Samar Elkhateb • Nicole Moens • Mickael Aoun • \\ Jean Marie Nogaret
}

Received: 18 March 2008 / Accepted: 9 July 2008 /Published online: 6 August 2008

(C) Springer-Verlag 2008

\begin{abstract}
Actinomycosis is a chronic suppurative granulomatous disease caused by the Gram-positive bacteria Actinomycoses israelii. This infection rarely involves the pelvis where the main etiological risk factor is the use of an intra-uterine contraceptive device. We report on a case of pelvic actinomycosis which mimicked ovarian neoplasia both clinically, radiologically, and surgically. The final diagnosis was made based on the histopathology study of the removed organ. The clinical picture is often nonspecific and may suggest ovarian neoplasia. First line treatment is essentially medical, involving prolonged treatment with antibiotics. Additional surgical treatment may occasionally be appropriate.
\end{abstract}

Keywords Actinomycoses israelii Pelvic actinomycosis . Ovarian neoplasia $\cdot$ Antibiotic therapy $\cdot$ Pelvic mass

\author{
S. Elkhateb \\ CHU Hassan II, Université sidi Mohamed ben Abdellah, \\ Fes, Morocco \\ S. Elkhateb $\cdot$ M. Aoun · J. M. Nogaret \\ Institut Jules Bordet, Université Libre de Bruxelles, \\ Brussels, Belgium \\ N. Moens \\ Clinique Louis Cathy, \\ Baudour, Belgium \\ S. Elkhateb $(\bowtie)$ \\ $\mathrm{n}^{\circ} 6$, rue $\mathrm{n}^{\circ} 30$ (ex rue Sharif Al-Idrissi), Hay Sâada $1^{\mathrm{er}}$, \\ Fes 30000, Morocco \\ e-mail: elkhatebsamar@yahoo.fr
}

\section{Introduction}

Actinomycosis is an indolent bacterial infection caused by anaerobic bacteria from the Actinomycetes family, which are saprophytic in nature and are usually found colonizing the mouth, the colon, and the vagina. The most frequently encountered species is Actinomycoses israelii [1, 2]. However, other types of Actinomycetes have occasionally been implicated, either alone or in synergy with other commensal bacteria, thus suggesting a possible polymicrobial origin to this infection. The key step in the pathogenesis of actinomycosis is the breakdown of the natural anatomical barriers, especially the mucosa, after surgery, trauma, or in the presence of a foreign body.

It is essentially a granulomatous disease with a dense fibrotic reaction which can readily take on the aspect of a tumor. It is encumbered with a high degree of morbidity and can be life threatening [3]. The clinical and radiological diagnoses are hard to make, even by experienced clinicians. Clinical presentation is highly variable, and the location can be orofacial $(50-60 \%)$, pulmonary $(20-25 \%)$, abdominal (15-20\%), and pelvic (5\%) [3, 4]. Actinomycosis can be detected in $8 \%$ to $20 \%$ of women carrying an intra-uterine device (IUD) [5]. The clinical picture is highly variable and often dramatic, leading the surgeon to intervene and propose mutilating surgery.

In this article, we report on a new case of abdominopelvic actinomycoses, which is presented as advanced ovarian cancer, and where the correct diagnosis was only made postoperatively on histopathology.

Case report

A 39-year-old mother of two was referred to our institution for management of suspected ovarian malignancy with liver 
and lung metastases. The diagnosis had been made on the basis of clinical examination and clinical imaging. There was no relevant medical or surgical history. The patient had an IUD in situ for 5 years.

The patient gave a history of right iliac fossa pain of several months' duration which was cyclical in nature and accompanied by a mild fever during the menstrual period. There was no abnormal vaginal discharge. Clinical examination revealed several pelvic masses, and a magnetic resonance scan confirmed the presence of bilateral ovarian masses along with peritoneal carcinomatosis and retroperitoneal lymphadenopathy (Fig. 1). The tumor marker CA125 was raised at $51.76 \mathrm{IU} / \mathrm{ml}(\mathrm{N} . \mathrm{R} .<35 \mathrm{IU} / \mathrm{ml})$. A full blood count revealed a leucocytosis with a predominance of polymorphonucleocytes. The $\mathrm{C}$ reactive protein was found increased.

An exploratory laparotomy was performed with an aim to debulk the tumoral mass. It revealed two necrotic tumoral masses originating from the ovaries and adherent to the sigmoid colon, the caecum, and the terminal ileum. Bilateral salpingo-oophorectomy and an omentectomy were performed after careful dissection of the tumoral mass off the bowel. In light of severe inflammation and advanced surgical staging of the tumor, the hysterectomy and lymphadenectomy were not attempted.

The histopathology was characterized by the presence of multiple abscesses fused together to form a mass, with numerous colonies of actinomycoses in the form of sulfur grains in an inflammatory infiltrate rich with foamy

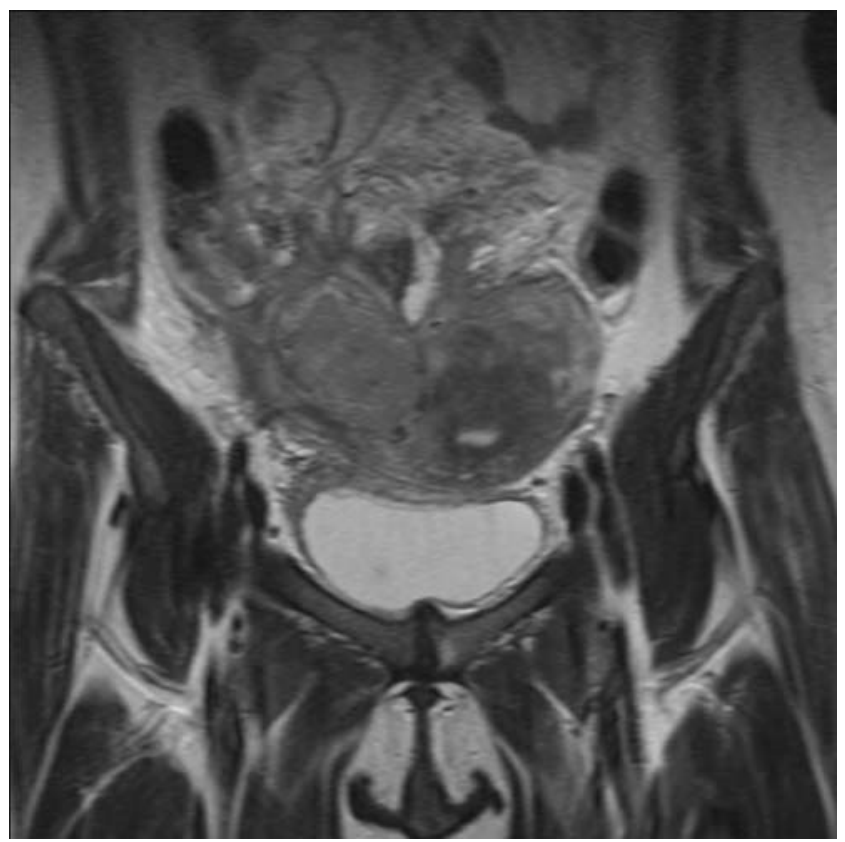

Fig. 1 A magnetic resonance scan showing bilateral ovarian masses displacing the uterus, with peritoneal carcinomatosis and retroperitoneal lymphadenopathy. Frontal section

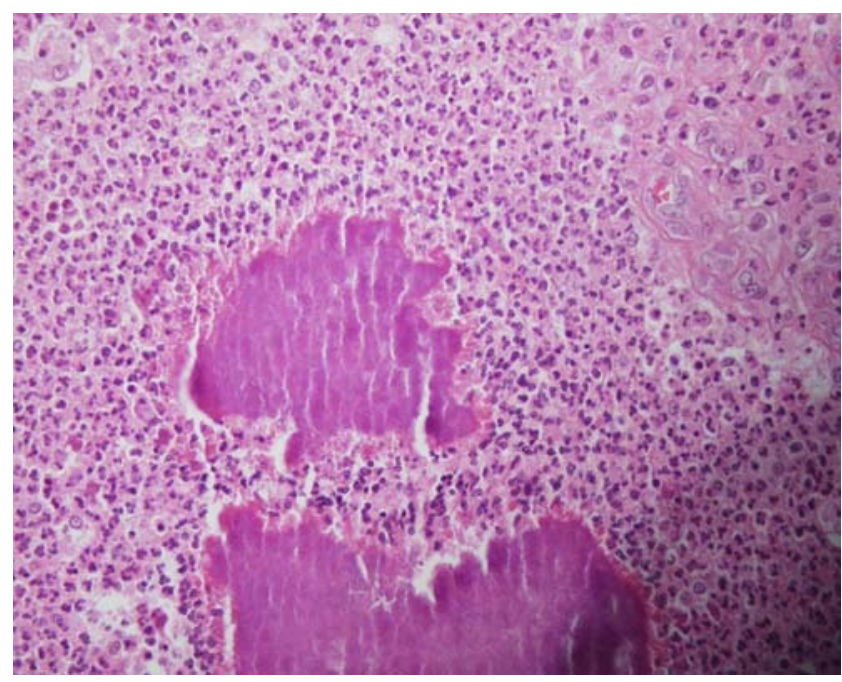

Fig. 2 Histological section showing a sulfur granule in an inflammatory exudate

macrophages and fibrin. The tissues examined were dilated, congested, and covered in fibrinous exudate rich in white blood cells. There was no sign of malignant degeneration (Fig. 2).

The post-operative course was punctuated by numerous complications in the short and medium term. A digestive fistula declared itself on the fourth post-operative day. The patient was managed medically, with parenteral feeding, somastatin, gastric prophylaxis, and a combination of antibiotics to be continued for a minimal period of 6 months (co-amoxiclav, 4-quinolones, pipericillin, and tazobactam). A nasogastric tube was sited and the gastric contents were aspirated under close clinical and biological surveillance. The IUD was removed and sent for microbiological analysis. The culture was negative. A gastrograffin transit study was inconclusive. The patient was returned to theater for assessment of the supposed fistula but no macroscopic fistula was found. A good clinical and biological response to the proposed medical treatment was noted with a reduction in the intestinal secretions. The patient was allowed to return home with out-patient follow up. After about 7 weeks, and in the context of lack of compliance with the antibiotic treatment and the patient's psycho-social problems, she presented with symptoms of intestinal subocclusion and a biological inflammatory syndrome. An urgent CT scan showed a pelvic abscess at the extremity of the pelvic drain. A laparotomy confirmed the diagnosis. Lavage and drainage were performed. Post-operatively, the patient was treated with the identical medical regime as during the first post-operative period, and the patient was kept nil by mouth with parenteral nutrition via a central line. There was steady improvement of the clinical condition with reduction in both abdominal drainage and intestinal secretions. Continued treatment with co-amoxiclav 
$875 \mathrm{mg}$ tds for 6 months was proposed and accepted by the patient.

\section{Discussion}

Reputed to be the worst-diagnosed disease since it was discovered by Israel in 1879 [6], the current reported case shows that this assertion remains true even nowadays. The clinical presentation, on the one hand, is non-specific and microbiological identification, on the other hand, is technically complicated: the clinical presentation is non-specific and the culture of the organism requires direct, rapid inoculation under strict anaerobic conditions. The culture then takes several days. A pelvic magnetic resonance scan is a useful alternative for making the diagnosis [7]: the characteristic abscess formation can be best seen in $\mathrm{T} 1$ sequences after gadolinium injection [8]. However, even at nuclear magnetic resonance scan in this case, the typical dense fibrosis of lesion and the little abscess formation were signs of a tumor. Perhaps, the determining element which would have to suggest actinomycoses is the presence of an IUD for 5 years. Indeed, pelvic actinomycoses has been linked to IUD use by several authors. Also, $88 \%$ of pelvic localizations are described in women whose IUD has not been changed for at least 3 years [9]. The disease process provoked by the presence of the IUD can progress, often silently, even after IUD removal [8]. The severity of the disease is affected by the quantity of proteolytic enzymes secreted by the bacteria, the presence of associated anaerobic bacteria, and the immune status of the host. This explains the wide variation in clinical severity which may range from chronic asymptomatic carriage to fulminant forms [4]. The natural evolution of these pseudo-tumoral masses or actimycetomata can be either towards abscess formation with risk of rupture, fistula formation and pelvic or more generalized peritonitis on the one hand, or spread to neighboring and distant organs on the other hand. Pelvis and abdominal forms are difficult to diagnose since the clinical presentation is so non-specific and variable. The presentation can simulate a neoplastic process when faced with an ill-defined and fixed pelvic mass or a colic or gynecological inflammatory process when faced with a clinical picture of infection and inflammation. Frozen histology sections are useful to provide a rapid diagnosis and avoid unnecessarily aggressive surgery. Pelvic actinomycoses can cause fever and pelvic pains and cause a general malaise with signs of low-grade infection. These symptoms, even present in the patient, were not quite discriminatory between neoplasia and a chronic infection as actinomycoses. It can also present as a pelvic mass. Extension to neighboring organs, fistula formation, and ureteric compression do occur but are rare [3]. Hematog- enous spread is even less common but explains the presence of hepatic lesions secondary to abdominopelvic lesions [10].

In fact, the diagnosis is most often made by the histological examination of the excised mass: the presence of characteristic clusters of organisms in a polysaccharide/ protein matrix known as "sulfur granules" is pathognomonic. These granules measure $0.25-1.00 \mathrm{~mm}$ in diameter, and resemble foliage of filaments whose ends are swollen like drumsticks. They stain positive for Gram, GomoriGrocott and PAS. Four cases have been reported where the diagnosis was made on a sample obtained by needle aspiration biopsy of the mass under either ultrasound or CT scan guidance, thus allowing primary surgery to be avoided $[11,12]$. Laparoscopy may be useful in diagnosis since it allows surgical biopsy as suggested by Barres et al. [13]. However, it is not recommended in the pseudotumoral forms of pelvic actinomycoses when an ovarian cancer for example is diagnosed [14].

Penicillin $\mathrm{G}$ is considered the treatment of choice. Other antibiotics such as clindamycin, rifamycin, or tetracyclines are also highly effective. In major forms of the disease, penicillin $\mathrm{G}$ is employed with a total daily dose of $10-20$ million units by the intravenous route for 4-6 weeks, followed by oral penicillin for 4-12 months. Initially, due to the delay in microbiological diagnosis, the presence of a digestive fistula, and arguments in favor of a polymicrobial etiology, we favored in this case the combination of amoxicillin with clavulanic acid instead of penicillin alone. The radical surgery described in most of the cases in the literature was carried out because of the lack of a firm preoperative diagnosis. Surgical treatment is certainly justified however in a case complicated by fistula or abscess formation [4]. Actinomycoses is particularly recognizable by the dense fibrosis around the infection site, which not only makes surgery more challenging but also hinders the antibiotics attaining adequate tissue levels, especially where the fibrosis has formed loculations. It is important to ensure that, along with the antibiotic therapy, the source of the infection is also removed, whether it is an IUD or an orodental foyer.

The prognosis depends on prompt treatment. It is therefore important to perform cervicovaginal swabs on women at risk because of IUD use [9]. This examination allows diagnosing Actinomycoses quite early. Treatment is therefore simpler and results are better.

\section{Conclusion}

The diagnosis of pelvic actinomycoses is difficult because it is not a well-known disease. It is rare and can be serious. A missed diagnosis can lead to life-threatening illness or 
unnecessary radical surgery. It must be excluded in cases of prolonged IUD carriage associated with general malaise, sub-febrile states, and/or pelvic or abdominal masses. The clinical presentation may suggest either a neoplastic or an inflammatory process. The treatment should consist of conservative surgery to obtain a firm histological diagnosis and to drain any collections, and long-term penicillin therapy.

\section{References}

1. Atallah D, Salet Lizee D, Van Den Akker M, Gadonneix P, Tranbaloc P, Villet R (1999) Actinomycose génitale: difficultés, diagnostiques et thérapeutiques. À propos de trois cas. Ann Chir 53:291-296

2. Garbin O, Hummel M, Camus E, Dellanbach P (1994) Actinomycose pelvienne: intérêt du diagnostic pré-opératoire. J Gynecol Obstet Biol Reprod 23:395-401

3. Abdelmoula-Marzouki S, Ben Brahim E, Ben Othmen M, Bouden S, Falfoul A, Chatti-Dey S (2003) Actinomycose tubo-ovarienne pseudotumorale fistulisée au côlon. Ann Chir 128:719-721

4. Bittar I, Cohen Solal JL, Cabanis P (2001) L'actinomycose abdominopelvienne. Ann Chir 126:494-496
5. Kayikcioglua MF, Akgula A, Haberala A, Demirb OF (2005) Actinomyces infection in female genital tract. Eur J Obstet Gynecol Reprod Biol 118:77-80

6. Israel J (1878) Neue Beobachtungen auf dem Gebiete der mykosen des Menschen. Virch Arch Path Anat 74:15-53

7. Hawnaur JM, Reynolds K, McGettigan C (1999) Magnetic resonance imaging of actinomycosis presenting as pelvic malignancy. Brit J Radiol 72:1006-1011

8. Bazot M, Davenne C, Benzakine $\mathrm{Y}$, Boudghène $\mathrm{F}$, Bigot JM (1997) Tubo-ovarite actinomycosique. Apport de l'angioscanner pelvien. J Radiol 78:513-516

9. Iwasaki M, Nishikawa A, Akutagawa N, Fujimoto T, Teramoto M, Kudo R (2003) A case of ovarian actinomycosis. Infect Dis Obstet Gynecol 11:171-173

10. Michel P (2004) Actinomycose pelvienne révélée par une pelvipéritonite. Ann Chir 129:96-99

11. Longchamps E, Vessieres A, Fabre M (2000) Comment la ponction guidée à l'aiguille fine peut éviter une chirurgie mutilante. À propos d'un cas d'actinomycose abdominopelvienne. Ann Pathol 20:145-149

12. Lee YCh, Min D, Holcomb K, Buhl A, DiMaio T, Abulafia O (2000) Computed tomography guided core needle biopsy diagnosis of pelvic actinomycosis. Gynecol Oncol 79:318-323

13. Barres A, Criscuolo JL, Vilde F, Taurelle R (1990) Actinomycose tuboovarienne. Rev FR Gynecol Obstet 85:479-482

14. Michel P (2004) Pelvic actinomycosis revealed by a pelvic peritonitis. Ann Chir 129:96-99 